$\mathrm{DE}$

M E D I C I N A

T R O P I C A L

$\mathrm{DE}$

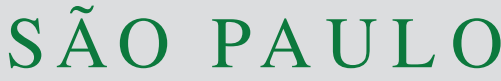

JOURNAL OF THE SÃO PAULO INSTITUTE OF TROPICAL MEDICINE

${ }^{1}$ Instituto Nacional de Medicina Genómica, Departamento de Genómica Poblacional, Genómica Computacional e Integrativa, Ciudad de México, Mexico

2Universidad Nacional Autónoma de México, Facultad de Medicina, Unidad de Investigación en Medicina Experimental, Centro de Medicina Tropical, Ciudad de México, Mexico

${ }^{3}$ Instituto Nacional de Medicina Genómica, Unidad de Secuenciación, Ciudad de México, Mexico

${ }^{4}$ Universidad Nacional Autónoma de México, Facultad de Medicina, Unidad de Investigación en Medicina Experimental, Laboratorio de Inmunoparasitología, Ciudad de México, Mexico

${ }^{5}$ Instituto Tecnológico y de Estudios Superiores de Monterrey, Escuela de Ingeniería y Ciencias, Monterrey, Nuevo León, Mexico

Correspondence to: Ingeborg Becker Hospital General de México, Dr. Balmis 148, Col. Doctores, 06726, Ciudad de Mexico, Mexico

Tel: +525556232674

Email: becker@unam.mx

Received: 19 December 2019

Accepted: 22 April 2020

\section{Relevance of epidemiological surveillance in travelers: an imported case of Leishmania tropica in Mexico}

\author{
Edith Araceli Fernández-Figueroa ${ }^{\circledR 1,2}$, Sokani Sánchez-Montes ${ }^{\circledR}$ 2, Haydee \\ Miranda-Ortíz ${ }^{\circledR 3}$, Alfredo Mendoza-Vargas ${ }^{\circledR 3}{ }^{3}$, Rocely Cervantes-Sarabia ${ }^{4}$, \\ Roberto Alejandro Cárdenas-Ovando ${ }^{(15}$, Adriana Ruiz-Remigio ${ }^{4}$, Ingeborg \\ Becker $^{(1), 4}$
}

\section{ABSTRACT}

We report the case of a patient with cutaneous leishmaniasis who showed a rapidly progressing ulcerative lesion after traveling to multiple countries where different Leishmania species are endemic. Diagnosis of Leishmania tropica, an exotic species in Mexico was established by using serological and molecular tools.

KEYWORDS: Leishmania tropica. Molecular epidemiology. Local cutaneous leishmaniasis. Travel medicine.

\section{INTRODUCTION}

Human cutaneous leishmaniasis is a zoonotic emerging tropical disease caused by 20 species of flagellated protozoa of the genus Leishmania, generating 150,000 new human cases per year, that are distributed across 98 countries throughout the Old World and the New World ${ }^{1-3}$. Most of the Old World cases are caused by Leishmania aethiopica, Leishmania infantum, Leishmania major and Leishmania tropica, whereas in the New World they are mostly caused by Leishmania amazonensis, Leishmania braziliensis, Leishmania mexicana, Leishmania naiffi and Leishmania panamensis $^{3,4}$.

Many of the species cause ulcers with indurated borders, which are indistinguishable between the species, thereby making the differential diagnosis very complicated $^{5-7}$. Yet a species-specific diagnosis is crucial since some Leishmania species generate visceral or mucocutaneous forms of the disease ( $L$. infantum and L. braziliensis respectively $)^{3,8}$. Currently, no morphological parameters exist that permit to distinguish between Leishmania species ${ }^{6,7}$. The increasing worldwide travel, ecoturism and displacement of migrants are affecting the distribution and incidence of patients with leishmaniasis, especially in the Middle East countries where L. major and L. tropica are the main pathogenic species ${ }^{9-11}$. In Mexico, most of the cutaneous leishmaniasis cases are caused by L. mexicana ${ }^{12,13}$. Yet, isolated reports of other species such as L. amazonensis, L. braziliensis and L. infantum/ chagasi have also been registered ${ }^{13}$. The surveillance of Leishmania species that circulate in a region is essential to alert health authorities of the introduction of exotic species that can affect vulnerable groups and also helps to establish the degree of dissemination or flow of these species within non-endemic regions ${ }^{2-4}$.

We report the case of a patient with LCL who showed a rapidly progressing ulcerative lesion after travelling to multiple countries where different Leishmania 
species are endemic. Diagnosis of leishmaniasis was established serologically and by smears, yet PCR enabled us to establish that the patient was infected by Leishmania tropica, an exotic species in Mexico. We highlight the importance of including a species-specific diagnosis by PCR to establish the Leishmania species and the possible infection site in travellers exposed to multiple species.

\section{CASE REPORT}

A 35-years-old male patient, originally from Russia, attended the Dermatology Service of the General Hospital in Mexico due to two ulcerative skin lesions with a semi-dry appearance, active borders, one of which was in the lower left pre-atrial region and the other in the dorsal region of the left forearm (Figure 1A). The patient referred having travelled to different Middle Eastern countries (Israel, Turkey), and to the Balkan region (Armenia, Georgia) and Russia, between May and June 2019. In August of the same year, he detected red and indurated papules, leading him to seek medical attention in San Cristobal de las Casas, located in the Mexican State of Chiapas, in late August. Cryotherapy with liquid nitrogen was applied to the lesions, leading to their temporary reduction in size. After a renewed flare-up of the lesion edges, the patient was attended at the Tropical Medicine Center (UNAM/Hospital General de Mexico), where he was clinically diagnosed as having localized cutaneous leishmaniasis (LCL). For the confirmation, Giemsa-stained smears of the lesion and ELISA tests for L. braziliensis, L. infantum/chagasi, L. major and L. mexicana were carried out ${ }^{14}$. Additionally, an aspirate of the lesion was performed and the recovered material was grown in NNN medium (Novy-MacNeal-Nicolle) and in 199 Hank's culture medium (Cat. 12350039, Gibco,
Burlington, Ontario, Canada), supplemented with $10 \%$ decomplemented fetal bovine serum- FBS (Cat. 16000044, Gibco, Burlington, Ontario, Canada). The quickly growing promastigotes were used for species identification. To this end, promastigote DNA was extracted with DNeasy Blood \& Tissue kit (QIAGEN Inc., Hilden, Germany) following the manufacturer's guidelines. For the PCR analysis, a 589 bp segment of the alanine aminotransferase gene (ALAT), a $350 \mathrm{bp}$ of the heat shock protein $70(h s p 70)$ and $300 \mathrm{bp}$ of the internal transcribed spacer $1(\text { ITSI })^{15,16}$ were amplified using $25 \mu \mathrm{L}$ QIAGEN Master mix (QIAGEN Inc., Hilden, Germany), $1 \mu \mathrm{L}(10 \mu \mathrm{M})$ of each primer (ALAT: Fwd 5'-GTGTGCATCAACCCMGGGAA3'and Rvs 5'-CGTTCAGCTCCTCGTTCCGC-3'; Hsp70: Fwd 5'-GGACGAGATCGAGCGCATGGT-3' and Rvs 5'-TCCTCCGACGCCTCCTGGTTG-3'; ITS1: Fwd 5'-CTGGATCATTTTCCGATG-3' and Rvs 5'-TGATACCACTTATCGCAC-3' ), 2-3 $\mu \mathrm{L}$ of extracted DNA and bi-distilled sterile water in a final volume of $50 \mu \mathrm{L}$. Additionally, L. mexicana DNA and bi-distilled sterile water were used as positive and negative controls, respectively. We followed the amplification conditions previously reported ${ }^{15,16}$. The PCR products were run on a $1 \%$ agarose gel stained with Smartglow (Cat. E4500PS, Accuris Instruments, Edison, NJ, USA). The PCR products were purified using the Agencourt AMPure XP kit (Cat. A63882, Beckman Coulter, Brea, CA, USA). Amplicons were sequenced using the BigDye Terminator v3.1 Cycle Sequencing Kit (Cat. 4337455, Thermo Fisher, Waltham, MA, USA). The sample was purified by the BigDye XTerminator (Cat. 4376486, Thermo Fisher, Waltham, MA, USA) prior to loading on the ABI 3730xL DNA analyzer (Thermo Fisher, Waltham, MA, US). Sequencing was carried out at the Sequencing Unit of the National Institute of Genomic Medicine.
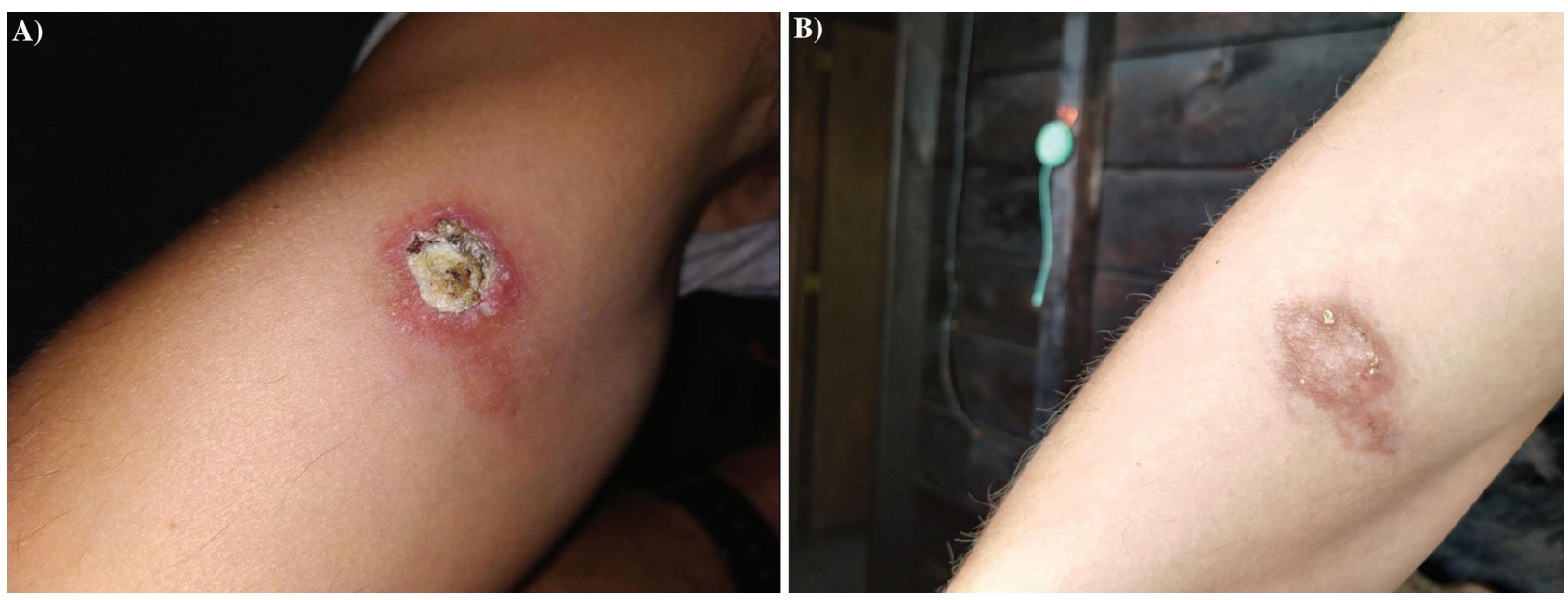

Figure 1 - Photographs of the ulcer on the forearm: A) active injury; B) injury after the glucantime treatment. 
Chromatograms were visualized and a consensus sequence was generated using the Chromas software (version 5, Technelysium, South Brisbane, QLD, Australia). The consensus sequence was compared to reference sequences deposited in GenBank using the BLASTn tool (National Center for Biotechnology Information, Bethesda, MD, USA). Sequences of validated Leishmania species were downloaded and a global alignment was conducted using the Clustal W algorithm (Informer Technologies, Inc., Dallas, US) with the software Bioedit (Informer Technologies, Inc., Dallas, US). Finally, a Maximum Likelihood phylogenetic reconstruction was carried out using the Tamura-three parameters model with invariant sites (I) with 10,000 Bootstrap replicates in Mega 6.0. Gaps were excluded from the analysis. Sequences were deposited in GenBank under the accession numbers MT157311, MT157312, and MT140349.

The Giemsa-stained smears of the lesion showed abundant amastigotes within macrophages. The ELISA analyzed the patient's serum revealing some degree of cross reactivity between four Leishmania species: L. braziliensis, L. infantum/chagasi, L. major and L. mexicana, yet higher antibody levels were recorded for L. major and L. mexicana antigens (Table 1). The isolated parasites were used for the PCR evaluation and a successful amplification of the three genes was achieved (Figures 2A, 2B and 2C). Using the BLAST analysis, these consensus sequences were $99 \%$ (538/544 bp), 100\% (323/323 bp), and 100\% (277/277 bp) identical to the corresponding sequences of the ALAT (MH455379), hsp70 (LN907846), and ITS1 (FN677341), genes respectively, of L. tropica from Israel and Russia. For the phylogenetic analyses, we only included fragments of 540, and $320 \mathrm{bp}$ of the ALAT, and $h s p 70$ genes, respectively. The Maximum Likelihood analysis confirmed that the parasite recovered from the patient corresponded to L. tropica (Figures 2D and 2E). The patient was treated with intralesional applications of $1 \mathrm{~mL}$ of glucantime per lesion at 5-day intervals with outpatient monitoring for 4 weeks. The case resolved favorably, the lesions healed adequately and the patient was discharged (Figure 1B).

Table 1 - The mean optical densities (OD) recorded in ELISA test for each Leishmania species.

\begin{tabular}{lcccc}
\hline Species & OD & Cut point & Result & Interpretation \\
\hline Leishmania braziliensis & 0.501 & 0.249 & 0.252 & Borderline \\
Leishmania infantum/chagasi & 0.356 & 0.178 & 0.178 & Borderline \\
Leishmania major & 0.435 & 0.191 & 0.244 & Positive \\
Leishmania mexicana & 0.548 & 0.204 & 0.344 & Positive \\
\hline
\end{tabular}
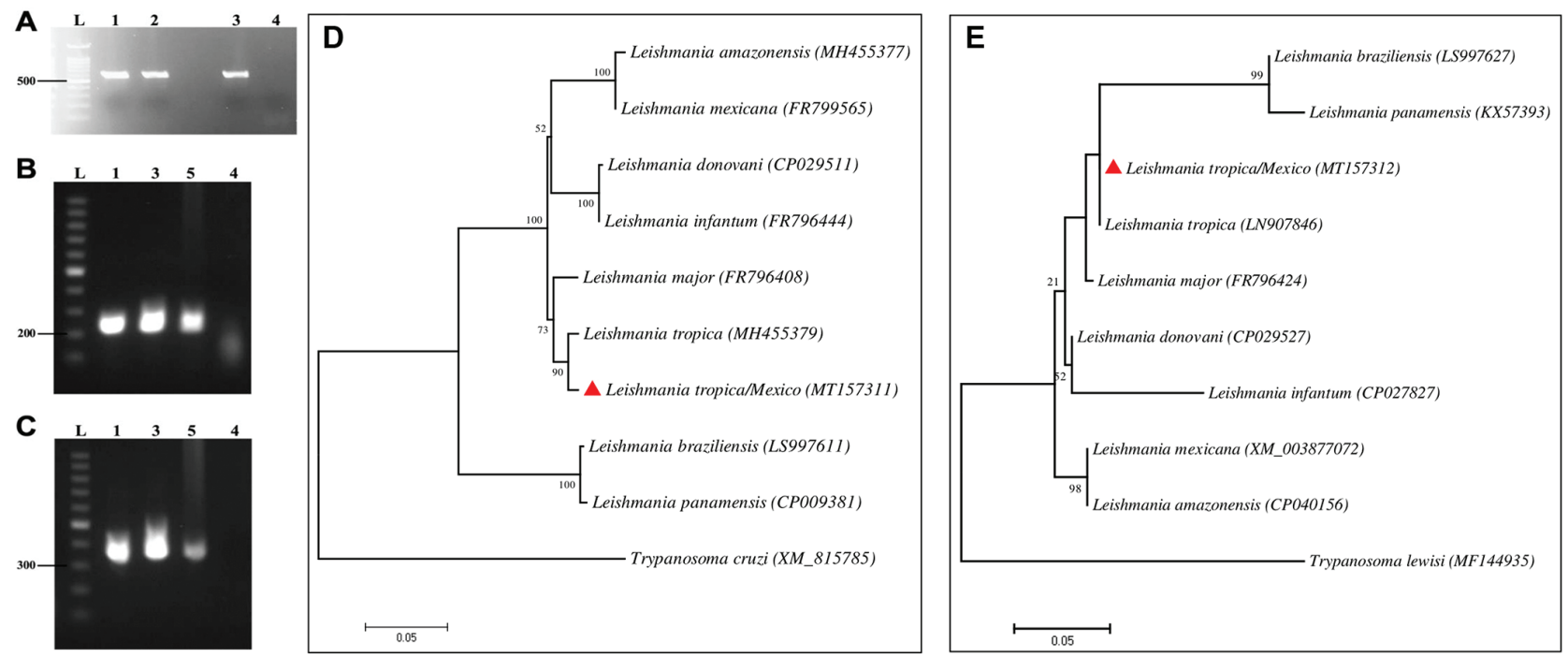

Figure 2 - Molecular identification of Leishmania tropica: A) ALAT. PCR products of parasites isolated from the patient in lines 1 and 2 (3 and $2 \mu \mathrm{L}$, respectively), Leishmania mexicana positive control (line 3), Nuclease-free water as the negative control (line 4). $\mathrm{L}=\mathrm{Ladder} 100 \mathrm{pb}$; B) $h s p 70-\mathrm{C})$ ITS1. PCR products of parasites isolated from the patient in lines 1 and 3 ( 3 and $2 \mu \mathrm{L}$, respectively), positive control (line 5), and negative control (line 4); D) Maximum Likelihood phylogenetic trees generated using the Tamura threeparameter distance model with Invariant sites $(+\mathrm{l})$ with partial sequences of the ALAT gene, and E) Hasegawa-Kishino-Yano with Gamma distribution ( $+\mathrm{G}$ ) of the $h s p 70$ gene from several members of the genus Leishmania. Bootstrap values greater than 50 are indicated on the nodes. The sequences generated in this study are indicated with red triangles. 


\section{DISCUSSION}

The molecular epidemiology of human imported cases of leishmaniasis is lacking in Mexico ${ }^{12,13}$. To the best of our knowledge, this case represents the first record of LCL caused by Leishmania tropica reported in Mexico. This species is registered mainly in the Middle Eastand Russia, representing one of the most widely distributed species causing cutaneous leishmaniasis in the Old World ${ }^{5,7,17,18}$. The incidence of this pathogen has increased in the urban areas of Israel and also in regions with armed conflicts in the Middle East ${ }^{16}$. Although the patient had visited several areas where $L$. tropica is endemic, he had also visited various States of Mexico, endemic for L. mexicana ${ }^{1,13}$. Thus, a species-specific diagnosis was mandatory. This was achieved by using molecular tools that enabled to detect the presence of L. tropica, an exotic microorganism in America. The rapid and correct detection of an infecting Leishmania species is important for establishing the correct treatment and the clinical management of the patients ${ }^{5,7}$. Furthermore, the correct identification of Leishmania species in travelers helps alerting health authorities on the presence of a novel species within the country and enable sanitary measures to avoid the establishment and distribution of new sources of Leishmania transmission in non-endemic regions, where sand flies are able to transmit the parasite ${ }^{8,9}$. In Middle East countries, Phlebotomus sergenti, is the main vector of $L$. tropica $^{19}$. Although in America there are no representatives of the genus Phlebotomus, in Mexico 52 sand fly species have been recorded, four of them are confirmed vectors of other Leishmania species and whose transmission capacity of L. tropica is unknown ${ }^{12}$.

Furthermore, environmental aspects such as climate change, growing urbanization, socio-economic development, tourism and migrations are changing the epidemiology of leishmaniasis $^{9-11}$. This highlights the need of establishing the molecular diagnostic as an essential tool for the active surveillance to avoid the emergence of outbreaks of exotic parasites.

\section{ETHICAL APPROVAL}

The current report was approved by the Ethics and Research Committee of the Medical Faculty of the UNAM (Universidad Nacional Autónoma de México), FMED/CI/ JMO/129/2017). The patient signed a written consent for the publication of the case.

\section{FUNDING}

This work was supported by grants from the CONACyT
221405, PAPIIT IN211418 and PAPIIT IN209314.

\section{REFERENCES}

1. Pigott DM, Bhatt S, Golding N, Duda KA, Battle KE, Brady OJ, et al. Global distribution maps of the leishmaniases. eLife. 2014;3:e2851.

2. Akhoundi M, Kuhls K, Cannet A, Votýpka J, Marty P, Delaunay $\mathrm{P}$, et al. Historical overview of the classification, evolution, and dispersion of Leishmania parasites and sandflies. PLoS Neg1 Trop Dis. 2016;10:e0004349.

3. Alvar J, Vélez ID, Bern C, Herrero M, Desjeux P, Cano J, et al. Leishmaniasis worldwide and global estimates of its incidence. PLoS One. 2012;7:e35671.

4. Ashford RW. The leishmaniases as emerging and reemerging zoonoses. Int J Parasitol. 2000;30:1269-81.

5. de Vries HJ, Reedijk SH, Schallig HD. Cutaneous leishmaniasis: recent developments in diagnosis and management. Am J Clin Dermatol. 2015;16:99-109.

6. Scorza BM, Carvalho EM, Wilson ME. Cutaneous manifestations of human and murine leishmaniasis. Int J Mol Sci. 2017;18:E1296.

7. Van der Auwera G, Dujardin JC. Species typing in dermal leishmaniasis. Clin Microbiol Rev. 2015;28:265-94.

8. Solomon M, Sahar N, Pavlotzky F, Barzilai A, Jaffe CL, Nasereddin A, et al. Mucosal leishmaniasis in travelers with Leishmania braziliensis complex returning to Israel. Emerg Infect Dis. 2019;25:642-8.

9. Hodiamont CJ, Kager PA, Bart A, de Vries HJ, van Thiel PP, Leenstra $\mathrm{T}$, et al. Species-directed therapy for leishmaniasis in returning travellers: a comprehensive guide. PLoS Negl Trop Dis. 2014;8:e2832.

10. Carvalho BM, Maximo M, Costa WA, Santana AL, Costa SM, Costa Rego TA, et al. Leishmaniasis transmission in an ecotourism area: potential vectors in Ilha Grande, Rio de Janeiro State, Brazil. Parasit Vectors. 2013;6:325.

11. Rehman K, Walochnik J, Mischlinger J, Alassil B, Allan R, Ramharter M. Leishmaniasis in Northern Syria during civil war. Emerg Infect Dis. 2018;24:1973-81.

12. González C, Rebollar-Téllez EA, Ibáñez-Bernal S, Becker-Fauser I, Martínez-Meyer E, Peterson A, et al. Current knowledge of Leishmania vectors in Mexico: how geographic distributions of species relate to transmission areas. Am J Trop Med Hyg. 2011;85:839-46.

13. Torres-Guerrero E, Quintanilla-Cedillo MR, Ruiz-Esmenjaud J, Arenas R. Leishmaniasis: a review. F1000Res. 2017;6:750.

14. Escalona-Montaño AR, Pérez-Montfort R, Cabrera N, Mondragón-Flores R, Vélez-Ramírez DE, Gómez-Sandoval $\mathrm{JN}$, et al. Proteín phosphatase PP2C in the flagellum of Leishmania major: cloning and characterization. Parasitol Open. 2017;3:e15. 
15. Marco JD, Barroso PA, Locatelli FM, Cajal SP, Hoyos CL, Nevot $\mathrm{MC}$, et al. Multilocus sequence typing approach for a broader range of species of Leishmania genus: describing parasite diversity in Argentina. Infect Genet Evol. 2015;30:308-17.

16. Graça GC, Volpini AC, Romero GA, Oliveira Neto MP, Hueb M, Porrozzi R, et al. Development and validation of PCR-based assays for diagnosis of American cutaneous leishmaniasis and identification of the parasite species. Mem Inst Oswaldo Cruz. 2012;107:664-74.

17. Uchida S, Oiso N, Sanjoba C, Matsumoto Y, Yanagihara
S, Annoura T, et al. Cutaneous leishmaniasis caused by Leishmania tropica in Israel. J Dermatol. 2018;45:e240-1.

18. Ghatee MA, Taylor WR, Karamian M. The geographical distribution of cutaneous leishmaniasis causative agents in Iran and its neighboring countries, a review. Front Public Health. 2020;8:11.

19. Jancarova M, Hlavacova J, Volf P. The development of Leishmania tropica in sand flies (Diptera: Psychodidae): a comparison of colonies differing in geographical origin and a gregarine coinfection. J Med Entomol. 2015;52:1378-80. 